\title{
Smart ambulance using IoT for blood transfer facilities
}

\author{
Mohamed A. Torad ${ }^{1}$, Yahia H. Hossamel-din² \\ ${ }^{1}$ Higher Technological Institute, Department of Electrical Engineering, Egypt \\ ${ }^{2}$ Future University in Egypt (FUE), Department of Mechanical Engineering, New Cairo, Egypt
}

\begin{tabular}{l}
\hline \hline Article Info \\
\hline Article history: \\
Received Feb 18, 2020 \\
Revised Oct 23, 2020 \\
Accepted Dec 5, 2020 \\
\hline
\end{tabular}

Keywords:

Android

Best shortest path algorithm Internet of Things

\begin{abstract}
In case of an accident occurrence, ambulance try to transport wounded and injured people to the nearest hospital, but if the injured persons need an urgent blood transfer, the emergency reception (ER) can check the availability of the needed blood type and quantity. If it is available, the emergency reception accepts the injured person, but if it is not available, the reception reroutes the ambulance to the nearest hospital which have the required blood type and quantity. So, a system created that save that wasted time to save injured people life as possible. So, we create a system that save that wasted time to save injured people life as possible. So, the main contribution in this paper is selecting and determining the nearest hospital that own the proper blood types to save injurjed people lives using dijkstra algorithm. In this study, we use an android application at the ambulance which permit the paramedic to login and select the required blood type and quantity needed after determining the injured person's blood type and the android application automatically determine the nearest hospital using the best shortest path algorithm (SPA) after checking the database and generate a pin code for the paramedic to deliver it with injured person to the ER and get the needed blood. This process will consequently decrease the used blood quantity from the database.
\end{abstract}

This is an open access article under the CC BY-SA license.

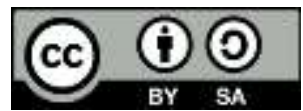

\section{Corresponding Author:}

Mohamed A. Torad

Department of Electrical Engineering

10th of Ramadan City, Egypt

Email: Mohamed.torad@gmail.com

\section{INTRODUCTION}

The road networks at each country considered as a sign of nation developments. Every single day, countries around world establish new roads in addition to maintain existing roads to accommodate population rising around the world. As a result, there are a lot of accidents which happen every day on the roads. Many reasons are responsible for it such as driver's fault, bad road, and pedestrian mistakes.

Along the past 15 years, the rate of road deaths has stayed constant at about approximately 1.35 million people per year because of road traffic crashes. Road traffic crashes cost most countries 3\% of their gross domestic product. More than half of all road traffic deaths are vulnerable road users: pedestrians, cyclists, and motorcyclists. Road traffic injuries are the leading cause of death for many children and young adults aged 5-29 years. about 20 to 50 million of people are suffering of a non-fatal injury but incurring a disability because of their injuries [1]. Many researchers' discuses road traffic and death rates from many prospective [2-7]. Traffic death rate can be decreased if injured persons reach at hospital in the proper time. Injured people send to the nearest hospital to the accident location by ambulances. At the hospital, some critical cases need intensive medical care with immediate blood transfer, sometimes the accidant nearest hospital don't own the blood types need, this condtion can lead to fatal consequences. 
Technologies such as short-range wireless communications, radio-frequency identification (RFID) and wireless sensor networks (WSN) have permitted the Internet to dive into embedded computing [8-11]. The internet of things (IoT) becomes a known communication paradigm that focuse connecting various kinds of devices to the Internet, in order to concatinate data acquired by remotely control, sensors, monitor environments, appliances and machines, buildings, and vehicles, and so on [12-17]. In this work, the designed system proposed to shorten the time and distance needed to transfer injured people from the accident location to the nearest proper hospital (i.e. which contain the required blood types for injured people). System is composed of two main parts: the database which contain all hospitals blood bags types and quantities, data acquired from sorting system located at each hospital listed at the system database and an android application with the paramedic at the ambulance. After that, application will select the nearest hospital according to the ambulance current location and shows the best shortest path to the desired hospital using the map. The android application checks the database, lists all hospitals have the required blood types and quantites. After that, application will select the nearest hospital according to the ambulance current location and shows the best shortest path to the desired hospital using the map. This proposed system expect to decrease time needed to deliver the accident injured persons to the nearest and proper hospital for them, which will save them lives.

Many literature reviews discuss similar problems from different aspects. Rajeshwari S. propose a system for smart traffic control system which allow to emergency vehicles including ambulance to cross traffic smoothly. PIC MC used with the aid of RFID tag and ZigBee module to check approaching of ambulance toward the traffic. If so, then traffic will switch to allow the ambulance to pass quickly [18]. Ran Zhang and Bo Zeng, propose two-stage robust optimization (RO) models to design a credible ambulance system related to absence of the ambulances, with and without the ambulance relocation. to preserve the surpass service coverages under various considerations [19]. R. Marques, et al. created an optimization model for selecting the best ambulance bases location, model consider many case study constrains (e.g. traffic condition, immediate availability of ambulance, distance to the emergency place in addition to the nature of the emergancey condition). This model suggest that the current bases location are not sufficient [20]. In [21] proposed a system to detect an accident occurrence using the builtin smartphone sensors and decrease the time needed to inform an accident occurrence by using the nearest fog node to inform the accident occureance and its location to the nearest hospital to provide an immediate assistant for the accident victums. Menghan Jia, et al. proposed a system that facilitate bracelets. To transmit a signal when abnormal action carry out by sending proper data to the hospital by using Petri net model of distributed resource allocation based on cloud medical system [22]. In [23] proposed a system for traffic monitoring and alert notification based on MongoDB and OpenGTS, this system is useful for drivers of critical rescue vehicles such as ambulances. Deepali A, et al. created a traffic control android application to clear all traffic in the ambulance way to the hospital. Android application was linked to traffic control trough database [24]. Hooman S. and Rongbo Z. were suppling the ambulance robot with an automated external defibrillator to help people whom suffering from sudden cardiac arrest [25]. Several sensors such as laser scanner, ultrasonic and camera are used to produce ambulance robot (AMBUBOT) and facilitates various manual and automatic functions to save people lives. Omkar U, et al. used various sensors such as ECG blood pressure and heart rate sensor to send vital parameters to the hospital from the ambulance through cloud which will operating simultaneously the traffic signals using GPRS message [26]. Poonam G, et al. created an application which shows the Patient in need of an ambulance and informs the proper hospitals with the patient case, which consequently permit the hospital to send the ambulance with the proper medically equipment [27]. The route from the hospital to the patient location was detected on the google map using the GPS built-in smartphone.

Also, many literature reviews the dijkstra algorithm which used to shorten the time and distance for the ambulance to reach the proper hospital D.C. Lee, verify the correctness of a modified Dijkstra's algorithm that calculates the shortest-delay path in networks with deterministically time-varying paths [28]. The main contribution of this paper is decreasing the elapsed time for the ambulance to reach to the desired hospital (i.e. own the needed blood types and bags) using dijkstra algorithm. The rest of paper is divided into three sections; Secion 2 which discuss research method; followed by results and analysis section; to conclusion is the final section

\section{RESEARCH METHOD}

The system consists of two parts: database and android application. The database links the ambulance application with the hospitals application whereas the hospitals listed at the database only have the hospital applications. Every hospital listed at the database has a sorting system which can increase or decrease a certain blood type according to the blood admission or taken from the hospital respectively. At ambulance application, the system can save the injured persons lives by detecting the nearest hospital 
equipped with the required blood type and quantity. The application uses firebase and android tools. Using the android application, the paramedic will have the authority to create an account to access the database via sign up icon. When the paramedic signs up in the application, the paramedic data were stored at the database with a unique ID (UID). If so (i.e. paramedic has an account), paramedic can access the database by signing in the Android application located at the ambulance using his name, password and email. Table 1 shows the login activity algorithm whereas the paramedic can access the database using his username and password and enter the blood types and quantities at the corresponding field of the android application. Application checks the database and lists all the proper hospitals which have the required blood types and quantities and detects their locations with respect to the ambulance location which determined using GPS located at the ambulance, then selects the nearest hospital location using the best shortest path algorithm (e.g. Dijkstra's algorithm). Table 2 shows the ambulance current location algorithm whereas the map is shown in the ambulance Android application, shows both the ambulance location which was determined using the ambulance GPS and the predefined target hospital location from the database and the routing path. In addition, PIN code is generated to the paramedic that will be used upon arriving at the desired hospital reception to acquire the blood type and quantity needed and decrease them from the database using Android application located at the sorting system in the hospital automatically. Increment and decrement process done using and quick response (QR) scanning manually in the hospital Android application. Figure 1 and 2 shows the hospital Android system flowchart and the ambulance Android system flowchart respectively.

The database tool used is the firebase. The Firebase Realtime database is a cloud-hosted database. Data is stored as JSON tree and synchronized in realtime to all the connected clients. When a cross-platform application is linked to the android application SDKs, all the clients will share the same Realtime database instantly and automatically will receive the updates of the latest data.

Table 1. Login activity algorithm

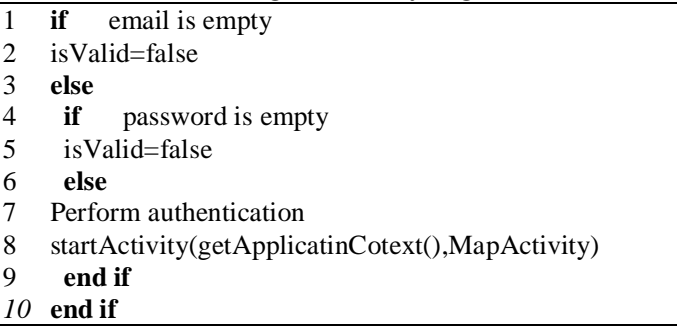

Table 2. Ambulance current location algorithm
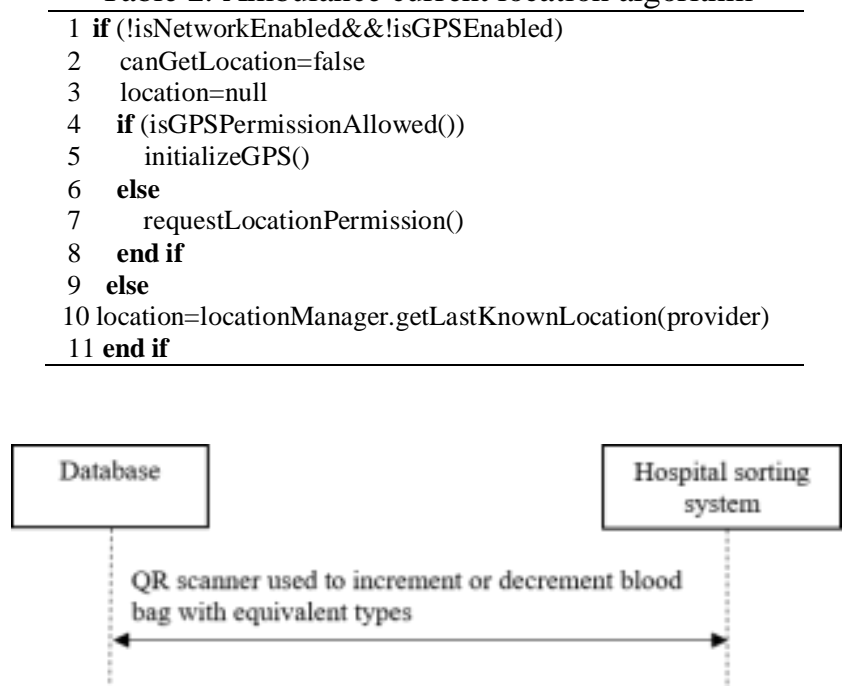

Figure 1. The hospital android system flowchart 


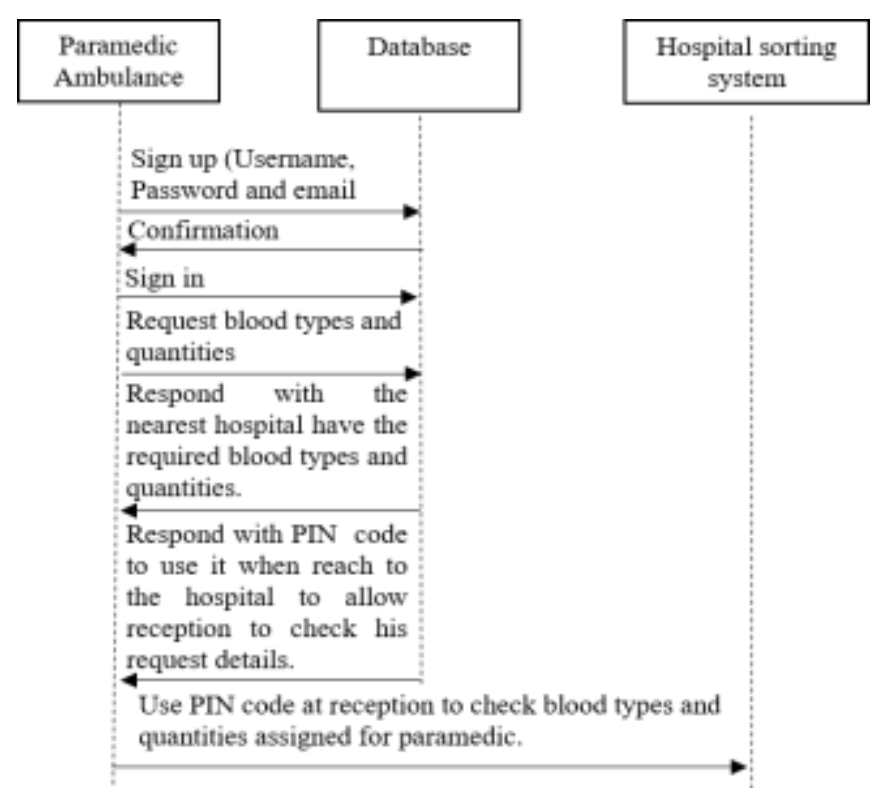

Figure 2. The ambulance android system flowchart

\section{RESULTS AND DISCUSSION}

The paper focuses on the link between the android application and the firebase, which used to store and save numbers and accounts and consequently allow the following operations to carry out:

a) Creation of a new account.

b) Paramedic' sign up.

c) Paramedic' sign in.

d) Paramedic' request of blood bags and quantities.

e) Increment or decrement of blood quantities with the equivalent types from the Database.

f) Checking the quantity available of certain blood type.

The firebase is used as it's an efficient and a low-latency solution for mobile applications that require synced states across clients in real time database. Figure 3 shows the log in page of the ambulance android application where the paramedic can choose the option: create new account; for the first time. If the paramedic already has an account, he uses his username (i.e. email) and password to login to the database.

When the paramedic logged into the system successfully, the blood request page will be appeared as shown in Figure 4. The database states all the hospitals owing the requested blood types and quantities to the application with their relative distance from the ambulance using the map dialog algorithm as shown in Table 3. Then, the application choses the nearest hospital based on the best shortest path algorithm (i.e. Dijkstra Algorithm) using google map application programming interface (API) and the path will be shown on the map with the estimated time to reach to the desired hospital as shown in Figure 5.

At the accident location, when the paramedic request blood types and quantities, a PIN code will be generated which will be used when the paramedic reaches the hospital. The recpitionist at the hospital check the needed blood type and quatity using the PIN code generated at the accident location, as shown in Figure 6. At the hospital, a two separated process are taking place. The first one is the PIN code verification as shown in Figure 6 while the second is the updates of the quantities of each blood type either by increment or decrement at the database using the android application. So, a unique QR patch was assigned for each blood type.

Table 3. Map dialog algorithm

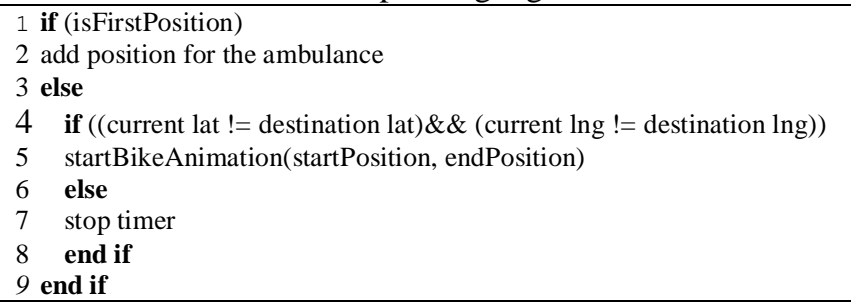




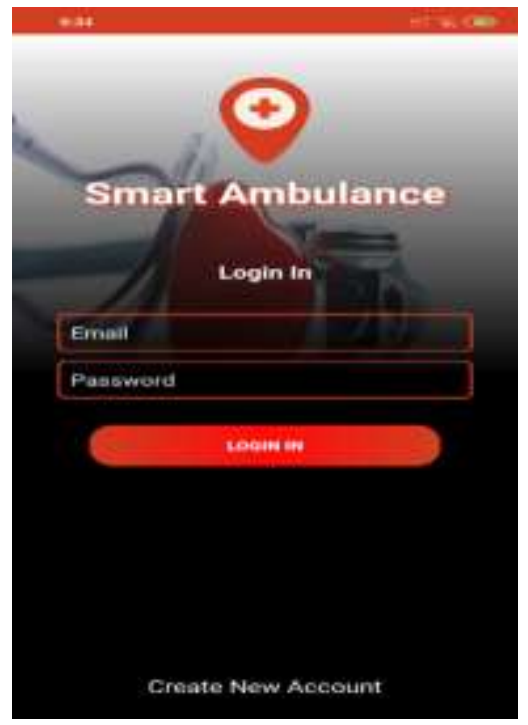

Figure 3. Paramedic login page

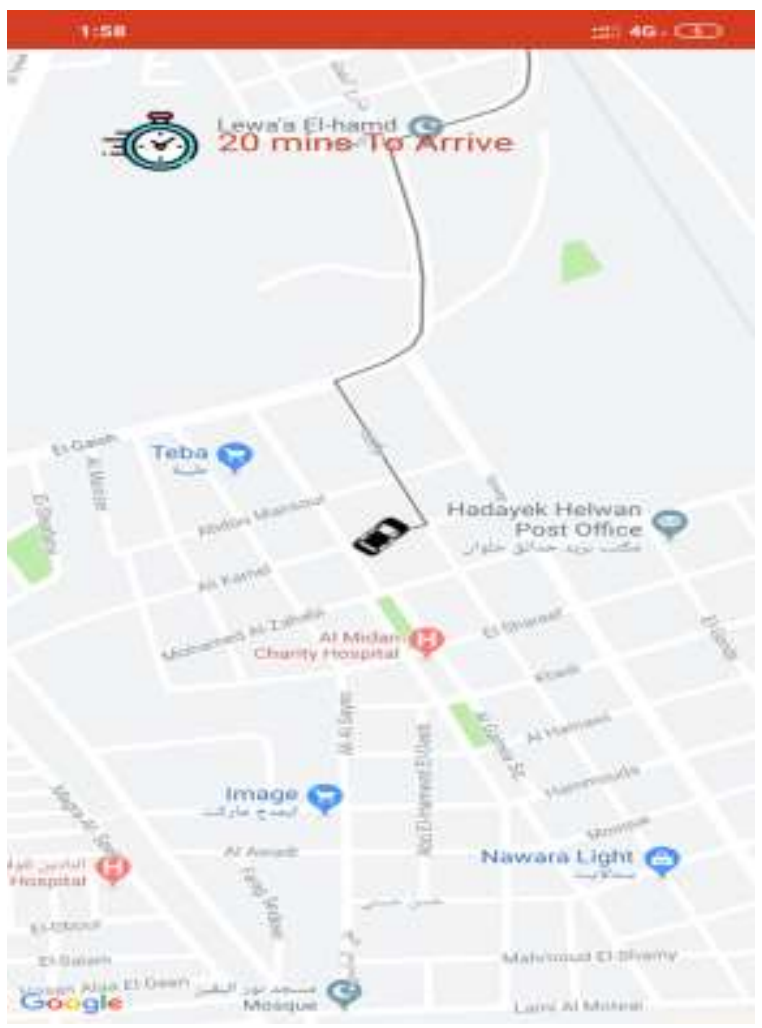

Figure 5. The google map showing the shortest path to the desired hospital

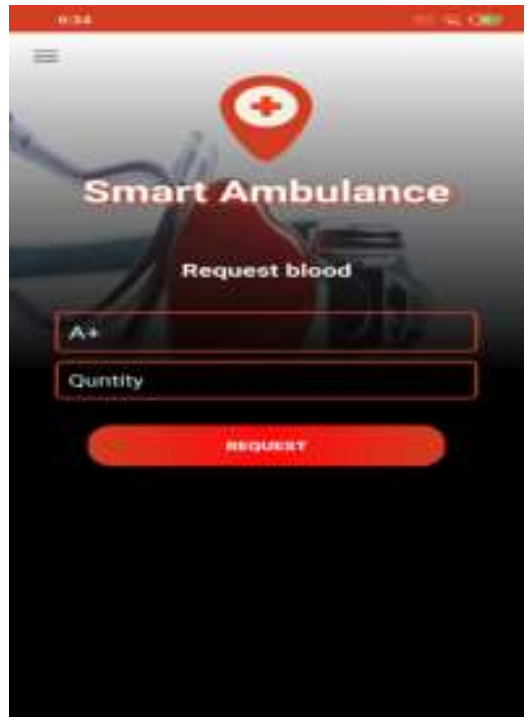

Figure 4. Blood request screen

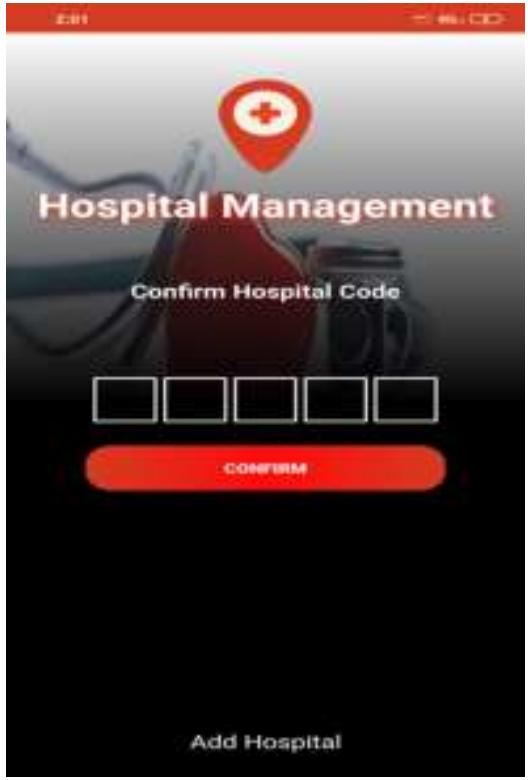

Figure 6. The PIN code check page

When the hospital gets a new blood bags, the receptionist adds them to the database by selecting the add option then he scans their QR path via the hospital Android application which consequently increase the added quantity to their corresponding types. On the other hand, when the receptionist pulls a quantity of a certain blood type for any surgery or operation using the PIN code of the paramedic, he selects pull option then scans their QR path via the hospital Android application which consequently decrease them from their corresponding types. 


\section{CONCLUSION}

This work creates a new system which saves accident injured people lives and minimizes the probabilities of death due to certain blood bags' leakage in the hospital by selecting the best nearest hospital with the proper blood types with respect to accident location. The amendment that can be added to this model, the blood reservation time model which will rescue more lives in certain hard conditions (e.g. earthquake, disasters). This model will allow the blood reservation from far away and subsequently will shadow the reserved quantities temporarily from the database for a certain time. The model will set according to a certain time for the reservation process, after that while the reservation will cancel automaticaly if the person requesting blood is not present. This model can integrated into an optimization model to take into consideration all emergency facilities (e.g. number of free ICU units, and suergery teams).

\section{REFERENCES}

[1] https://www.who.int/gho/road_safety/mortality/en/

[2] Unaiza Alvi, Muazzam A. Khan Khattak, Balawal Shabir, Asad Waqar Malik, Sher Ramzan Muhammad, "A Comprehensive Study on IoT Based Accident Detection Systems for Smart Vehicles," IEEE Access, vol. 8, pp. 122480-122497, 2020, doi: 10.1109/ACCESS.2020.3006887.

[3] Chaojie Ou, Fakhri Karray, "Enhancing Driver Distraction Recognition Using Generative Adversarial Networks," IEEE Transactions on Intelligent Vehicles, vol. 5, no. 3, pp. 385-396, 2020, doi: 10.1109/TIV.2019.2960930.

[4] Nuerzhegeti Aiyitibieke, Wenjun Wang, Nannan Wu, Ying Sun, Xuewei Li, Yueheng Sun, "An Empirical Study on High-Risk Driving Behavior to Urban-Scale Pattern in China,” IEEE Access, vol. 7, pp. 43654-43665, 2019, doi: 10.1109/ACCESS.2019.2906930.

[5] Sinnu Susan Thomas, Sumana Gupta, Venkatesh K. Subramanian, "Event Detection on Roads Using Perceptual Video Summarizationl," IEEE Transactions on Intelligent Transportation Systems, vol. 19, no. 9, pp. 2944-2954, 2017, doi: 10.1109/TITS.2017.2769719.

[6] Sachin Umesh Sharma, Dharmesh J. Shah, “A Practical Animal Detection and Collision Avoidance System Using Computer Vision Technique,” IEEE Access, vol. 5, pp. 347-358, 2017, doi: 10.1109/ACCESS.2016.2642981.

[7] Carlos Massera Filho, Marco H. Terra, Denis F. Wolf "Safe Optimization of Highway Traffic With Robust Model Predictive Control-Based Cooperative Adaptive Cruise Control," IEEE Transactions on Intelligent Transportation Systems, vol. 18, no. 11, pp. 3193-3203, 2017, doi: 10.1109/TITS.2017.2679098.

[8] Huifeng Wu, et al., "Dynamic Edge Access System in IoT Environment," IEEE Internet of Things Journal, vol. 7, no. 4, pp. 2509-2520, 2020, doi: 10.1109/JIOT.2019.2943696.

[9] Danyang Zheng, Chengzong Peng, Xueting Liao, Xiaojun Cao, "Toward Optimal Hybrid Service Function Chain Embedding in Multiaccess Edge Computing," IEEE Internet of Things Journal, vol. 7, no. 7, pp. 6035-6045, 2020, doi: 10.1109/JIOT.2019.2957961.

[10] Qiao Li, Ying Song; Boxin Du; Yu Shen; Yuan Tian, "Deep Neural Network-Embedded Internet of Social Computing Things for Sustainability Prediction," IEEE Access, vol. 8, pp. 60737-60746, 2020, doi: 10.1109/ACCESS.2020.2982986.

[11] Shishupal Kumar, Vijay Kumar Chaurasiya, "A Strategy for Elimination of Data Redundancy in Internet of Things (IoT) Based Wireless Sensor Network (WSN)," IEEE Systems Journal, vol. 13, no. 2, pp. 1650-16557, 2019.

[12] Ala Al-Fuqaha, Mohsen Guizani, Mehdi Mohammadi, Mohammed Aledhari, Moussa Ayyash "Internet of Things: A Survey on Enabling Technologies, Protocols, and Applications," IEEE Communications Surveys \& Tutorials, vol. 17, no. 4, pp. 2347-2376, 2015, doi: 10.1109/COMST.2015.2444095.

[13] Andreas Kamilaris, Andreas Pitsillides "Mobile Phone Computing and the Internet of Things: A Survey," IEEE Internet of Things Journal, vol. 3, no. 6, pp. 885-898, 2016, doi: 10.1109/JIOT.2016.2600569.

[14] Mohammad Abdur Razzaque, Marija Milojevic-Jevric, Andrei Palade, Siobhán Clarke "Middleware for Internet of Things: A Survey," IEEE Internet of Things Journal, vol. 3, no. 1, pp. Pp. 70-95, 2016, doi: 10.1109/JIOT.2015.2498900.

[15] Francesca Meneghello, Matteo Calore, Daniel Zucchetto, Michele Polese, Andrea Zanella, "IoT: Internet of Threats? A Survey of Practical Security Vulnerabilities in Real IoT Devices," IEEE Internet of Things Journal, vol. 6, no. 5, pp. 8182-8201, 2019, doi: 10.1109/JIOT.2019.2935189.

[16] Lalit Chettri, Rabindranath Bera "A Comprehensive Survey on Internet of Things (IoT) Toward 5G Wireless Systems," IEEE Internet of Things Journal, vol. 7, no. 1, pp. 16-32, 2020, doi: 10.1109/JIOT.2019.2948888.

[17] Xuyang Ding, Ying Xie, Pengxiao Li, Mengtian Cui, Jianying Chen "Image Steganography Based on Artificial Immune in Mobile Edge Computing With Internet of Things," IEEE Access, vol. 8, pp. 136186-136197, 2020, doi: 10.1109/ACCESS.2020.3010513.

[18] Rajeshwari S., Santhoshs Hebbar, Varaprasad Golla, "Implementing Intelligent Traffic Control System for Congestion Control, Ambulance Clearance, and Stolen Vehicle Detection," IEEE Sensors Journal, vol. 15, no. 2, pp. 1109-1113, Feb 2015.

[19] Ran Zhang and Bo Zeng, "Ambulance Deployment With Relocation Through Robust Optimization," IEEE Transactions On Automation Science And Engineering, vol. 16, doi: 10.1109/TASE.2018.2859349. 
[20] R. Marques, T. Queiroz, and R. Borges, "A Robust Model for the Bases Location and Ambulances Allocation Problem," IEEE Latin America Transactions, vol. 17, no. 11, pp. 1887-1893, Nov 2019, doi: 10.1109/TLA.2019.8986428.

[21] Bilal khalid, munam ali shah, saif ul islam, castren maple, Shafaq mussadiq, and suleman khan "Delay-Aware Accident Detection and Response System Using Fog Computing," IEEE Access, vol. 7, pp. 70975-70985, May 2019, doi: 10.1109/ACCESS.2019.2910862.

[22] Menghan Jia, Wangyang Yu, Xiaojun Zhai, Sangeet Saha "Modeling and Analysis of First Aid Command and Dispatching System of Cloud Medical System," IEEE Access, vol. 7, pp. 168752-168758, Nov 2019, doi: 10.1109/ACCESS.2019.2954451.

[23] Antonio Celesti, Antonino Galletta, Lorenzo Carnevale, Maria Fazio, Aime Ĺay-Ekuakille, Massimo Villari “An IoT Cloud System for Traffic Monitoring and Vehicular Accidents Prevention Based on Mobile Sensor Data Processing,” IEEE Sensors Journal, vol.18, no. 12, June 2018, doi: 10.1109/JSEN.2017.2777786.

[24] Deepali Ahir, Saurabh Bharade, Pradnya Botre, Sayali Nagane, and Mihir Shah, "Intelligent Traffic Control System for Smart Ambulance," International Research Journal of Engineering and Technology (IRJET), vol. 05, no. 06 June-2018.

[25] Hooman Samani and Rongbo Zhu "Robotic Automated External Defibrillator Ambulance for Emergency Medical Service in Smart Cities," IEEE Access, vol. 4, pp. 268-283, Jan-2016, doi: 10.1109/ACCESS.2016.2514263.

[26] Omkar Udawant, Nikhil Thombare, Devanand Chauhan, Akash Hadke, Dattatray Waghole JSCOE, and PUNE "Smart Ambulance System using IoT," International Conference on Big Data, IoT and Data Science (BID) Vishwakarma Institute of Technology, Pune, pp. 171-176, Dec 20-22, 2017, doi: 10.1109/BID.2017.8336593.

[27] Poonam Gupta, Satyasheel Pol, Dharmanath Rahatekar, and Avanti Patil "Smart Ambulance System," International Journal of Computer Applications, 2016.

[28] D.C. Lee "Proof of a modified Dijkstra's algorithm for computing shortest bundle delay in networks with deterministically time-varying links," IEEE Communications Letters, vol. 10, no. 10, Oct. 2006, doi: 10.1109/LCOMM.2006.051982.

\section{BIOGRAPHIES OF AUTHORS}

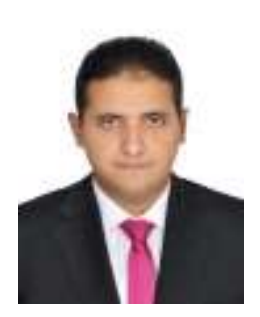

Mohamed A. Torad Received the B.Sc. degree in electrical engineering from Higher Technological Institute (HTI), in 2007. From 2008, he has been a research assistant at communication and electronics department, Higher Technological Institute, received his M.D. from Ain Shams University (ASU) of at 2013. and received his PhD degree from Ain Shams University (ASU) of at 2016. He still working at communication and electronics department from 2007 till now, working at Future University at Egypt (FUE) and supervises number of graduation projects at Culture and Science City. Work as reviwer at many conferances (e.g. International Conference on Microelectronics (ICM), IEEE International Multi-Conference on Systems, Signals \& Devices).

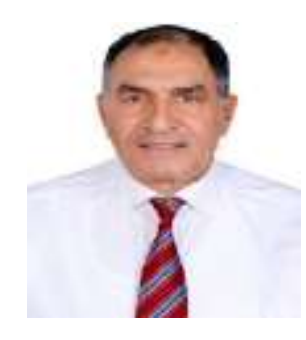

Yehia H. Hossamel-din is currently a professor and Chairman of the Mechanical Engineering Department; Future University in Egypt (FUE). Received his PhD from the University of Wisconsin-Madison, USA (1986), MSc and BSc from the Military Technical College (MTC); Cairo; Egypt (1977 \& 1970; respectively). He also worked as a professor and chairman of Mechatronics Engineering Deparment at Phyladelphia University (PU); Amman; Jordan (20012005), the Higher Technological Institute (HTI); Tenth of Ramadan City, Egypt (2006-2014) and the Military Technical College (1986-1996). He took part in many International Journals and conferences as an author, presenter, keynote speaker, reviewer, and organizer. He is the representative of Egypt on the international network for Research and Education in Mechatronics (REM). 